\title{
Effects of externally supplied BSA on ZR, IAA, ABA, GA of plant Cinnamomum camphora cutting seedlings and root ShaoHui Huang ${ }^{1}$ \\ ${ }^{1}$ School of Environmental Engineering, Xuzhou institute of technology, XuZhou 221008, China axhshui@163.com
}

\begin{abstract}
Proteins possible effects on roots have received little attention. Here, we investigated how externally supplied protein influences root formation of Cinnamomum camphora cuttings. Cultivation matrix was composed of vermiculite, perlite and river sand based on the proportion 2 : $2: 3$. Softwood cuttings were soaked and injected by the bovine serum albumin (BSA). The results demonstrated that the addition of low to intermediate concentrations of protein ( $\leqq 7.58 \mu$ M BSA) increased rooting rate, average number of lateral roots and average root length of cuttings. However, the rooting rate, the number and length of lateral root decreased at higher concentrations of BSA ( $\geqq 15.15 \mu$ M BSA). The treatment of injection was more effective than that of soaking. The endogenous hormones (ZR, IAA, ABA, GA) content of the cuttings rapidly decreased after cutting. On the different days after cutting, four endogenous hormones decreased to the lowest value respectively. Low to intermediate concentrations of BSA will alter the content and proportion of 4 endogenous hormones and be conducive to root development. But the higher concentrations of exogenous BSA changed the content and proportion of 4 endogenous hormones was not in favor of root formation.
\end{abstract}

\section{Keywords Externally supplied protein $\bullet$ Root $\bullet$ Endogenous hormone $\bullet$ Cutting $\bullet$ Cinnamomum} camphora

\section{Abbreviations: BSA (Bovine serum albumin); ZR (Zeatin riboside); IAA (Indole-3-acetic acid); NO (Nitric oxide); ABA (Abscisic acid); GA (Gibberellic acid).}

\section{Introduction}

The genetic component determines the fundamental morphology and blueprint of a plant's root system, whereas environmental cues shape root architecture by modifying the intrinsic genetic program $^{[1]}$. The nutritional status and type of nutrient determined the extent and type of changes in root system architecture ${ }^{[2]}$. A branching architecture dimension expresses root plastic responses to the environment ${ }^{[3]}$. Plant root development is highly responsive to changes in nitrate availability ${ }^{[4]}$. NO has a key role in signal transduction network in plants influencing the action of hormones ${ }^{[5]}$. During adventitious root formation in chrysanthemum cuttings, 3\% protein was related to nitrate metabolism and $7 \%$ of hormone related ${ }^{[6]}$. Ramie adapted to NPK deficiency by promoting the intracellular flow of carbon and $\mathrm{N}$, promoting the synthesis cysteine and related hormones and up regulating action protein to promote growth of the root system ${ }^{[7]}$. Genetic engineering of nitrogen assimilation has received considerable attention for its improvement of nitrogen use efficiency in crops ${ }^{[8]}$. Recently, molecular regulators in nitrate or ammonium sensing and signaling have been isolated, revealing common regulatory genes for the transport system and root development, as well as a strong connection between $\mathrm{N}$ and hormone signaling pathways ${ }^{[9]}$.

Less is known about the effects of organic nitrogen forms on root growth, because organic nitrogen has received less attention as $\mathrm{N}$ source. Historically, a reason is that only inorganic $\mathrm{N}$ and L-amino acids have been considered to be important to the $\mathrm{N}$ nutrition of terrestrial plants ${ }^{[10]}$ has 
largely replaced the focus on organic nitrogen. Unfertilized, cool-climate cropping, soil is dominated by soluble and bound amino acids ${ }^{[11]}$. Amino acids are also prevalent in sub/tropical agricultural soil ${ }^{[12]}$. Organic nitrogen compounds of intermediate molecular mass were detected in temperate agricultural soil ${ }^{[10]}$. The glutamine synthetase enzyme and the DNA binding with one finger transcription factor play important roles in the regulation of nitrogen metabolism that is essential for plant growth and development ${ }^{[13]}$.

The evidence that organic nitrogen elicits change to root growth is accruing. Plants grown in different $\mathrm{N}$ mixtures were similar in size, but those supplied with organic $\mathrm{N}$ displayed a significantly greater root fraction ${ }^{[14]}$. Reduced glutathione treatment promoted root formation in cuttings grown on both base- and auxin-supplemented media ${ }^{[15]}$. Under controlled environmental conditions, plant uptake of organic and inorganic $\mathrm{N}$ forms in the dominant desert shrub Larrea tridentata was quantified to assess the potential effects of elevated atmospheric $\mathrm{CO}_{2}$ on plant $\mathrm{N}$ uptake dynamics ${ }^{[16]}$. High concentrations of single amino acids such as glycine near complete inhibition of root growth, and single amino acids such as glutamine, amides, peptides or mixtures of organic $\mathrm{N}$ compounds increased root/shoot ratio and root elongation, adventitious root formation $^{[14]}$. NO production and amino acid metabolism are interesting questions waiting for further investigation ${ }^{[17]}$. The effects of BSA on root morphology appear to be independent from auxin and ethylene signaling pathways ${ }^{[18]}$. This suggests that, similar to inorganic nitrogen and low molecular mass, organic nitrogen, protein elicits responses in roots. Here, we evaluated how protein affected root architecture and plant endogenous hormone changes in dose-response experiments with BSA and growth medium composed of vermiculite, Perlite and river sand.

\section{Materials and methods}

\section{Plant material and growth conditions}

Cinnamomum camphora softwood cutting used in this study was collected from the campus of the Xuzhou Institute of Technology. Softwood cuttings were selected according to the length of 7-10 cm, diameter of 0.3-0.4 cm and 2-3 half leaf retention. The cuttings were soaked with 5 kinds of BSA concentration in Scheme 1 (see Table 1) and injected with accordingly BSA concentration in scheme 2 (100 $\mu \mathrm{L}$ microsyringe come from Shanghai JiaAn analytical instrument factory) (see Table 1), and were contrasted with sterile water. Cultivation matrix was composed of vermiculite, perlite and river sand based on the proportion $2: 2: 3$. The experiments were repeated 3 times, respectively.

Cultivation matrix was disinfected with $2 \% \mathrm{KMnO}_{4} 7 \mathrm{~d}$ before cutting. Be rinse with water 24h before cutting and drilled in the matrix when cutting. The line method was used when cutting. Be watered the cutting immediately after cutting. Using an automatic spray device to spray at intervals of $1 \mathrm{~h}$ from the beginning of the second day to keep the humidity of the garden at around $95 \%$. The $1000 \mathrm{mgL}^{-1}$ carbendazim solution was sprayed enough after cutting to prevent mildew and rot of cuttings. Thiophanate methyl solution was sprayed in 3 weeks after cutting. Compound fertilizer and glucose aqueous solution were foliar applied every $15 \mathrm{~d}$.

The small arch shed of $40 \mathrm{~cm}$ high was built by the use of bamboo in the cutting bed surface in time after cutting. It was closed cover with $2 \mathrm{~m}$ wide fresh agricultural films and compacted around with soil. The western agricultural film was open for ventilation45-55dafter cutting. 0.5\%-1.0\%compound fertilizer was fertilized 1 times per month after root again in the autumn.

The content variation of 4 kinds of endogenous hormones GA, IAA, ABA and ZR were detected by the use of ELISA (enzyme linked immunosorbent assay) in the rooting process. The 
result of determination was calculated by Logit curve method. The test instrument was an enzyme mark instrument (Biotek Elx800, OD accuracy: $<1 \%$ at 2 OD) imported from America.

\section{Statistical analysis}

Statistical analyses were performed using ANOVA (IBM SPSS Statistics21), Mapping analysis using GraphPad Prism6.

\section{Results}

\section{Rooting rate changes caused by BSA treatment}

The cuttings of $C$. camphora were soaked (scheme 1 in Table 1) or injected (scheme 2 in Table 1 ) by the use of 1.52 to $45.45 \mu$ MBSA. Cuttings were cut in the disinfected cultivation matrix in 2013 July and the rooting rate, root amount and root length were detected in 2014 February.

Table 1:C. camphora cutting treated with BSA

\begin{tabular}{cccccccc}
\hline \multicolumn{1}{c}{ soaking } & BSA & mg BSA & Numbers of & Scheme 2 & injection \\
time $(\mathrm{h})$ & $(\mu \mathrm{M})$ & per L & cutting & $\begin{array}{c}\text { BSA } \\
\text { quantity }(\mu \\
(\mu \mathrm{M})\end{array}$ & $\begin{array}{c}\text { mg } \\
\text { BSA } \\
\text { per L }\end{array}$ & $\begin{array}{c}\text { Numbers of } \\
\text { cutting }\end{array}$ \\
\hline 4 & 1.52 & 0.1 & 50 & 100 & 1.52 & 0.1 & 50 \\
4 & 7.58 & 0.5 & 50 & 100 & 7.58 & 0.5 & 50 \\
4 & 15.15 & 1 & 50 & 100 & 15.15 & 1 & 50 \\
4 & 22.73 & 1.5 & 50 & 100 & 22.73 & 1.5 & 50 \\
4 & 45.45 & 3 & 50 & 100 & 45.45 & 3 & 50 \\
4 & sterile & sterile & 50 & & & & \\
& water & water & & & & & \\
\hline
\end{tabular}

Whether soaking or injected by BSA, low concentrations of BSA significantly improved rooting rate of cuttings (Fig. 1A, B) and the high concentrations of BSA significantly decreased the rooting rate of cuttings. The effect on the rooting rate by the method of BSA injected is more significant than that of BSA soaking cuttings. The rooting rate of cuttings soaked by the concentration of $1.52 \mu \mathrm{M}, 7.58 \mu \mathrm{M}$ BSA was increased by $20.8 \%, 18.7 \%$ than the contrast respectively. While the same concentrations of BSA injected, the rooting rate was increased by 21.3\% and 20.7\% than the contrast respectively. Soaked by the higher concentration of BSA such as $15.15 \mu \mathrm{M}, 22.73 \mu \mathrm{M}$ and $45.45 \mu \mathrm{M}$, the rooting rate was decreased by $6.3 \%, 12.5 \%$ and $18.8 \%$ than the contrast respectively. Injected by the same concentrations, the rooting rate was decreased by $8.3 \%, 14.6 \%, 20.8 \%$ than the contrast accordingly.
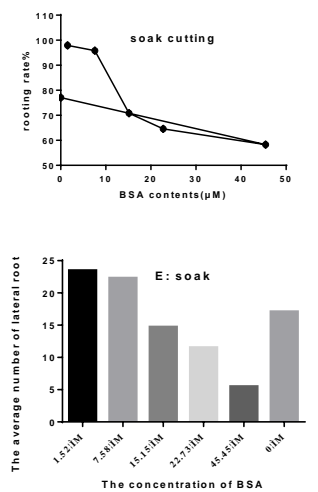
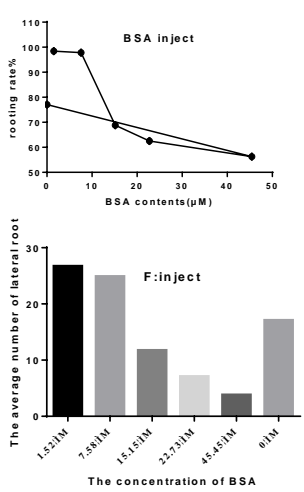
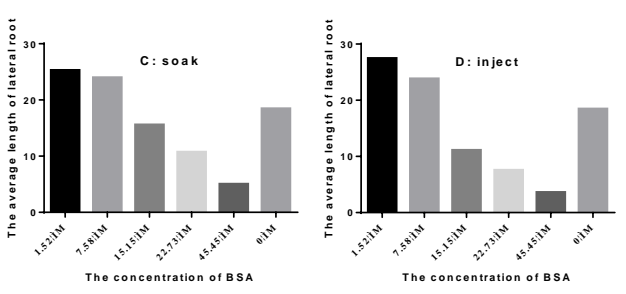

Fig.1 changes of rooting rate, average lateral root length and average lateral root number 


\section{BSA elicited the changes of average root length and rooting numbers of cutting}

Both of soaked and injected cuttings with low concentration of BSA (such as $1.52 \mu \mathrm{M}, 7.58$ $\mu \mathrm{M}$ ) increased the average number of lateral roots (Fig. 1E, F) and the average root length (Fig. 1C, D). However, the high concentration of BSA (such as $15.15 \mu \mathrm{M}, 22.73 \mu \mathrm{M}, 45.45 \mu \mathrm{M}$ ) decreased the average lateral root number and average root length. Whether low or high concentration of BSA is, the treatment of injection was more effective on the average number of lateral roots and average root length than that of soaking. Statistical analysis showed that soaked (Fig. 1C, E) and injected (Fig. 1D, F) treatment with $1.52 \mu \mathrm{M}$ BSA significantly increased (P < 0.05 ) the average number of lateral roots and average root length, and the treatments of $45.45 \mu \mathrm{M}$ BSA significantly decreased $(\mathrm{P}<0.05)$ the average number of lateral roots and the average root length.

\section{BSA elicited the changes of endogenous hormone in the course of cutting root development} The changes of endogenous $\mathrm{ZR}$ content

The endogenous ZR content of $C$. Camphora cutting declined rapidly after cutting7 days. The decreasing speed of endogenous ZR content was markedly correlated with concentration of BSA treatment. The lower the concentration of BSA treated cuttings ( $\leqslant 7.58 \mu \mathrm{M}$ BSA), the smaller the extent of decline. The higher the concentration of BSA treated cuttings ( $\geqslant 15.15 \mu \mathrm{M}$ BSA), the greater decreased degree of endogenous ZR content. The decreased degree by injecting cuttings was lower than soaked cuttings in the same low concentration of BSA. But higher concentration injected cuttings reacted more strongly than soaked cuttings. It was worth noticing that the different concentration of soaked and injected cuttings could result in endogenous ZR decreased at different rates. The content of ZR of the cuttings decreased to the lowest value on the twenty-first day, fourteenth day and twenty-first day, respectively, by the use of $1.52 \mu \mathrm{M}$ BSA soaked or injected cuttings, $7.58 \mu \mathrm{M}$ BSA soaked and 7.58 $\mu \mathrm{M}$ BSA injected accordingly. Whether soaked or injected cuttings by more than $15.15 \mu \mathrm{M}$ BSA, the content of ZR of the cuttings decreased to the lowest value on the fourteenth day. Then it began to decline after the rising in the twenty-eighth day. Thirty-five days later, the content of ZR of the cuttings treated by BSA less than $7.58 \mu \mathrm{M}$ began to decline.

\section{The changes of endogenous IAA content}

On the seventh day after cutting, the endogenous IAA content of the cuttings soaked and injected with various concentrations of BSA decreased to the lowest value respectively. But the magnitude of the decreased was different both in concentrations and ways. The content of endogenous IAA of the cuttings soaked and injected by $1.52 \mu \mathrm{M}$ BSA decreased less than treated with $7.58 \mu \mathrm{M}$ BSA. The higher concentrations of BSA, the greater magnitude decreased of the endogenous IAA content. It increased again to a higher value after 28 days, and then began to decrease. The new higher values of IAA content are lower than the initial values.

\section{The changes of endogenous GA content}

On the fourteenth day after cutting, the endogenous GA contents of the cuttings soaked and injected with various concentrations of BSA decreased to the lowest value respectively. But the magnitude of decreased was different both in concentrations and ways. The content of the endogenous GA of the cuttings injected decreased to a greater extent than that of soaked. Higher concentrations of BSA induced the content of the endogenous GA of the cuttings to decrease more than did of the lower concentrations BSA treatment. On the twenty-eighth day, the content of the GA of the cuttings soaked and injected with $7.58 \mu \mathrm{M}$ concentrations of BSA got new higher 
values than the initial values at the beginning of cutting. The content of the GA of the cuttings soaked with more than $15.15 \mu \mathrm{M}$ BSA was a lower value on the thirty-fifth day than the initial value at the beginning of cutting. It began to decrease them.

\section{The changes of endogenous ABA content}

After cutting, the content of the endogenous ABA of the cuttings soaked and injected with various concentrations of BSA decreased to a lower value on the seventh day. But the magnitude of the decreased was different both in concentrations and ways too. The content of endogenous ABA of the cuttings soaked decreased to a greater extent than that of injected. Higher concentrations of BSA induced the content of endogenous ABA of the cuttings to decrease more than did of the lower concentrations BSA treatment. On the fourteenth days, the content of ABA of the cuttings soaked and injected with various concentrations of BSA achieved a new higher value. At this moment, the content of ABA of the cuttings treated with $7.58 \mu \mathrm{M}$ concentrations of BSA got lower values than the initial values at the beginning of cutting, and the cuttings treated with more than $15.15 \mu \mathrm{M}$ concentrations of BSA achieved higher content of ABA than the initial ABA values. Later, the content of $\mathrm{ABA}$ of the cuttings treated with various concentrations began to decrease again. The higher concentrations of BSA, the faster decreased of content of ABA. Accordingly, the lower concentrations of BSA induced the content of ABA to decrease slowly.

\section{Discussion}

Here we quantified the responses of $C$. camphora cutting to the presence of high molecular mass organic nitrogen. We show that protein in the form of BSA affects plant morphology and plant endogenous hormone in a manner relating to treatment mode and the concentration of organic nitrogen. With sterile water as control, the results of our study agreement with the notion that roots exhibit measurable responses to organic nutrients irrespective of the plants' nutritional status ${ }^{[14]}$. In a concentrated and ways of treated dependent manner, BSA (i) alters rooting rate of cutting, (ii) modifies root architecture by influencing lateral root information, (iii) rapidly decreases the endogenous hormones (ZR, IAA, ABA, GA) content of the cuttings after cutting, and fluctuate with BSA concentration and ways of treated.

It is noteworthy that under severe $\mathrm{N}$ shortage, the lateral roots formation is almost completely absent ${ }^{[2]}$. Plant root development is highly responsive both to changes in nitrate availability in the rhizosphere ${ }^{[4]}$. The different plant species response to exogenous organic nitrogen is variable. For example, a positive effect of exogenous addition of a mix of three (Serine/Alanine/Glycine) or two (Serine/Alanine) amino acids on wheat root tolerance of oxygen deprivation was detected ${ }^{[19]}$. Reduced glutathione treatment promoted tomato seedling cuttings root formation ${ }^{[15]}$. Our experiment on the ornamental plants of C. camphora showed that the lower concentration of BSA is beneficial to the cuttings survived in adverse environments, and to the new roots formed as soon as possible. However, the highest concentrations of BSA are not conducive to the new root. Cutting propagation is the process that a part of the plant regenerates new individual by abscission from the parent in the face of adversity. We hypothesized that exogenous organic nitrogen in favor of plant adaptation to adverse adversity, and produce morphological and physiological response. Furthermore, this response is related to the organic nitrogen concentrations and application methods.

Hormones regulate plant growth and development in response to external environmental stimuli via complex signal transduction pathways, which in turn form complex networks of interaction. The plant root is a particularly useful system in which to study the complex role of 
plant hormones in the plastic control of plant development ${ }^{[20]}$. It has been demonstrated that all the stages of adventitious root formation are dependent on, or regulated by endogenous or exogenous auxin $^{[15]}$. Our experiment demonstrated that four kinds of endogenous hormone (ZR, IAA, ABA, GA) content decreased to a minimum in the short term as the plant suddenly suffered adversity. However, the exogenous organic nitrogen BSA will alter this change in different degrees. Lower concentrations of BSA will alter the content and proportion of 4 endogenous hormones in the $C$. camphora cuttings and be conducive to root development. But the higher concentrations of exogenous BSA will change the content and proportion of 4 endogenous hormones and was not in favor of root formation.

Four phytohormones we measured affecting on growth and development are well documented. The purified sedimentary humic acid action is functionally linked to increments in NO and IAA concentration in roots and also increases ethylene and ABA root concentration mediated by NO-IAA dependent pathways ${ }^{[21]}$. Lateral roots are crucial for the plasticity of root responses to the auxin and cytokinin balance in plants ${ }^{[22]}$. ABA significantly inhibited shoot growth and induced expression of OsKRP4, OsKRP5, and OsKRP6 ${ }^{[23]}$. The effect of $\mathrm{GA}_{3}$ treatments on the quantitative characteristics of Polianthes tuberose was investigated ${ }^{[24]}$. More recently, a strong connection between $\mathrm{N}$ and hormone signaling pathways have been revealed ${ }^{[9]}$. Our study adds the understanding that organic nitrogen has effects on the cuttings root formation, growth and endogenous hormone changes, highlights the necessity of application of organic nitrogen in garden plant operating and expands the existing knowledge that has been generated in cutting root formation test systems.

\section{Acknowledgments}

This study was supported by the Science and Technology Projects of Ministry of Housing and Urban-Rural Development of the people's Republic of China (No.2016-K2-005) and the Project of Natural Science Foundation of Jiangsu Province（No. BK20161164）

\section{References}

[1] Pacheco-Villalobos D, Hardtke CS, Natural genetic variation of root system architecture from Arabidopsis to Brachypodium: towards adaptive value, Philos Trans R Soc Lond B Biol Sci. 367(2012) 1552-1558.

[2] Benjamin D. Gruber, Ricardo F.H. Giehl, Swetlana Friedel, Nicolaus von Wirén, Plasticity of the Arabidopsis Root System under Nutrient Deficiencies, Plant Physiology. 163 (2013) (1) 161-179.

[3] Deliang Kong, Chengen Ma, Qian Zhang, Le Li, Xiaoyong Chen, Hui Zeng, Dali Guo, Leading dimensions in absorptive root trait variation across 96 subtropical forest species, New Phytologist. 203(2014) 863-872.

[4] Céline Contesto, Sandrine Milesi, Sophie Mantelin, Anouk Zancarini, Guilhem Desbrosses, Fabrice Varoquaux, Catherine Bellini, Mariusz Kowalczyk, Bruno Touraine, The auxin-signaling pathway is required for the lateral root response of Arabidopsis to the rhizobacterium Phyllobacterium brassicacearum, Planta, 232(2010) 1455-1470.

[5] Marcela Simontacchi, Carlos Garcı'a-Mata, Carlos G. Bartoli, Guillermo E. Santa-Marı'a, Lorenzo Lamattina, Nitric oxide as a key component in hormone-regulated processes, Plant Cell Rep. 32 (2013)853-866. 
[6] Ruixia Liu, Sumei Chen, Jiafu Jiang, Lu Zhu, Chen Zheng, Shuang Han, Jing Gu, Jing Sun, Huiyun Li, Haibin Wang, Aiping Song, Fadi Chen, Proteomic changes in the base of chrysanthemum cuttings during adventitious root formation, BMC Genomics. 14(2013) 919.

[7] Gang Deng, Li Jun Liu, Xin Yue Zhong, Cheng Ying Lao, Hong Yang Wang, Bo Wang, Cong Zhu, Fahad Shah, Ding Xiang Peng, Comparative proteome analysis of the response of ramie under N, P and K deficiency, Planta. 239(2014) 1175-1186.

[8] Wuling Lin, Ethan Hagen, Amy Fulcher, Michael Thomas Hren, Zong-Ming Cheng, Overexpressing the ZmDof1 gene in Populus does not improve growth and nitrogen assimilation under low-nitrogen conditions, Plant Cell Tiss Organ Cult. 113(2013) 51-61.

[9] Philippe Nacry, Eléonore Bouguyon, Alain Gojon, Nitrogen acquisition by the roots: physiological and developmental mechanisms ensuring plant adapted to a fluctuating resource, Plant Soil. 370 (2013) 1-29.

[10] Paul W. Hill1, Richard S. Quilliam, Thomas H. DeLuca, John Farrar, Mark Farrell, Paula Roberts, Kevin K. Newsham, David W. Hopkins, Richard D. Bardgett, David L. Jones, Acquisition and Assimilation of Nitrogen as Peptide-Bound and D-Enantiomers of Amino Acids by Wheat, PLoS ONE. 6 (4) (2011) e19220.

[11] Jämtgård S., Näsholm T., Huss-Danell K., Nitrogen compounds in soil solutions of agricultural land, Soil Biol. Biochem. 42(2010) 2325-2330.

[12] Holst J., Brackin R., Robinson N., Lakshmanan P., Schmidt S., Soluble inorganic and organic nitrogen in two Australian soils under sugarcane cultivation, Agric. Ecosyst. Environ. 155(2012) 16-26.

[13] Yilin Wang, Bing Fu, Lifeng Pan, Limei Chen, Xianhui Fu, Kunzhi Li, Overexpression of Arabidopsis Dof1, GS1 and GS2 Enhanced Nitrogen Assimilation in Transgenic Tobacco Grown Under Low-Nitrogen Conditions, Plant Mol Biol Rep. 31(2013) 886-900.

[14] Camila Aguetoni Cambui, Henrik Svennerstam, Linda Gruffman, Annika Nordin, Ulrika Ganeteg, Torgny Na“sholm, Patterns of Plant Biomass Partitioning Depend on Nitrogen Source, PLoS ONE. 6 (4) (2011) e19211.

[15] Jarosław Tyburski, Andrzej Tretyn, Glutathione and glutathione disulfide affect adventitious root formation and growth in tomato seedling cuttings, Acta Physiologiae Plantarum. 32(2010) 411-417.

[16] Virginia L. Jin, R. D. Evans, Elevated $\mathrm{CO}_{2}$ increases plant uptake of organic and inorganic N in the desert shrub Larrea tridentate, Oecologia. 163 (2010) 257-266.

[17] Anis M, Limami, Houssein Diab, Jérémy Lothier, Nitrogen metabolism in plants under low oxygen stress, Planta. 239(2014) 531-541.

[18] Thierry G. A. Lonhienne, Yuri Trusov, Anthony Young, Doris Rentsch, Torgny Näsholm, Susanne Schmidt, Chanyarat Paungfoo-Lonhienne, Effects of externally supplied protein on root morphology and biomass allocation in Arabidopsis, Nature: Scientific Reports Volume: 4, Article number: 5055 (2014) DOI: doi: 10.1038/srep05055

[19] Shingaki-Wells RN, Huang S, Taylor NL, Carroll AJ, Zhou W, Millar AH, Differential molecular responses of rice and wheat coleoptiles to anoxia reveal novel metabolic adaptations in amino acid metabolism for tissue tolerance, Plant Physiol, 156(2011)1706-1724

[20] Adriana Garay-Arroyo, María De La Paz Sánchez, Berenice García-Ponce, Eugenio Azpeitia1, Elena R. Álvarez-Buylla, Hormone symphony during root growth and development, Developmental Dynamics. 241 (12) (2012) 1867-1885. 
[21] Verónica Mora, Eva Bacaicoa, Roberto Baigorri, Angel M. Zamarreño, Jose' M. Garc1'a-Mina, NO and IAA Key Regulators in the Shoot Growth Promoting Action of Humic Acid in Cucumis sativus L.,J Plant Growth Regul. 33(2014) 430-439.

[22] Krome K., Rosenberg K., Dickler C., Kreuzer K., Ludwig-Müller J., Ullrich-Eberius C., Scheu S., Bonkowski M, Soil bacteria and protozoa affect root branching via effects on the auxin and cytokinin balance in plants, Plant Soil. 328 (2010)191-201.

[23] Meguro A., Sato Y, Salicylic acid antagonizes abscisic acid inhibition of shoot growth and cell cycle progression in rice,Sci. Rep. 4:4555(2014) DOI:10.1038/srep04555.

[24] Moazzam Hassanpour Asil, Zeynab Roein, Jafar Abbasi, Response of Tuberose (Polianthes tuberose L.) to Gibberellic Acid and Benzyladenine, Hort. Environ. Biotechnol.52 (1) (2011) 46-51. 